\title{
Health perceptions and treatment adherence in adults with cystic fibrosis
}

\author{
Janice Abbott, Mary Dodd, A Kevin Webb
}

\begin{abstract}
Background - Clinical and demographic variables are poor predictors of treatment adherence. This study therefore examined the relationships between the patients' perception of their cystic fibrosis and their reported adherence to physiotherapy, exercise, pancreatic enzyme and vitamin therapies.
\end{abstract}

Methods - Sixty adults with cystic fibrosis completed the Health Perception Scale, Health Locus of Control Scale, and the Manchester Adult Cystic Fibrosis Compliance Questionnaire.

Results - Reported adherence to physiotherapy, exercise, pancreatic enzyme and vitamin therapies was not influenced by patients' perceptions of their past, current and future disease severity, or their perceived susceptibility to recurrent infections. The greater their level of worry regarding their disease, the more likely they were to adhere to their physiotherapy $(p<0.002)$, pancreatic enzyme $(p<0.001)$ and vitamin $(p<0.001)$ regimens. No difference between the exercise adherent and non-adherent groups emerged regarding their level of worry. Compared with patients who believed that they were in control of their health, those who believed that their cystic fibrosis was controlled by others (family and health professionals) reported greater levels of adherence to their physiotherapy $(p<0.001)$, pancreatic enzyme $(p<0.001)$ and vitamin $(p<0.001)$ therapies. In contrast, patients who adhered to their exercise regimen believed that they were in control of their disease to a greater extent than those who did not $(p<0.003)$.

Conclusions - Worrying about cystic fibrosis and the perception of having little personal control over the disease facilitated treatment adherence. It is clear that patients perceive exercise therapy differently from other forms of treatment. (Thorax 1996;51:1233-1238)

Keywords: cystic fibrosis, adherence, health perceptions.

Increasing numbers of patients with cystic fibrosis are now surviving into adulthood. Over 6000 individuals in the UK have the disease, more than 2000 of which are adults. The national median age of survival is currently 25 years. ${ }^{1}$ The daily management involves a complex time-consuming range of treatments and self care. A significant improvement in survival has been attributed to earlier diagnosis, multidisciplinary specialist centre care, and more effective methods of treatment. Adherence to treatment may be an important factor in the successful management of the disease. As yet there are no conclusive data linking poor treatment adherence and progressive disease in cystic fibrosis, although it is commonly assumed that the consequences of poor adherence are infective exacerbations, disease progression, the costs of wasted drugs, increased outpatient visits and hospital admissions, and erroneous conclusions about the efficacy of the treatment.

Poor adherence is a common problem even with life threatening disorders. Five hundred and thirty seven studies reviewed by Sackett and Snow $^{2}$ in 1979 indicated that the range of adherence for long term preventative regimens was $33-94 \%$ with a mean adherence rate of $57 \%$. Similarly, for long term treatments the range was $41-61 \%$ with a mean adherence rate of $54 \%$. More recent work is consistent with these findings. Only $32 \%$ of patients with epilepsy were considered adherent with their treatment, ${ }^{3} 12-66 \%$ of children with asthma were non-adherent, ${ }^{4}$ and $58 \%$ of renal dialysis patients showed poor adherence. ${ }^{5}$ An overall adherence score, however, fails to recognise that patients may adhere to one aspect of their treatment but not to others. Previous findings from our clinic have demonstrated that reported adherence is treatment specific in cystic fibrosis, $53 \%$ of patients reported being adherent to their physiotherapy, $75 \%$ were considered to be exercising to a beneficial level, and $83 \%$ and $46 \%$ of patients reported that they always took their enzymes and vitamins, respectively. ${ }^{6}$ Work by Passero et al also supports the notion of treatment specific adherence.

The search for the determinants of poor adherence is encouraged in the hope of being able to predict those at risk and thereby design interventions for improving adherence. Demographic factors (age, sex, knowledge of disease, employment status) and clinical factors (disease severity, age at diagnosis, frequency of clinic visits) have been evaluated as possible predictors of adherence in cystic fibrosis with equivocal results. ${ }^{68-12}$

According to the Health Belief Model $^{13}$ patients' perceptions of their illness as serious, feelings of susceptibility, a belief that the treatment is beneficial, and a motivation or concern for one's health should theoretically predict adherence rather than clinically assessed disease severity. It has been shown that patients 
and physicians perceive disease severity and overall self care differently. From the physician's viewpoint patients underestimate the severity of the disease and overestimate self care. ${ }^{11}$ It is not surprising, therefore, that clinical measures of disease severity or physicians' assessments are not associated with adherence to treatments and advice. Patient-perceived severity of illness has been shown to predict adherence to penicillin and antihypertensive medication and to asthma and haemodialysis regimens. ${ }^{1415}$ When patients were asked their reasons for poor adherence the most consistent answers included busy time schedule and apathy and forgetfulness in addition to the commonly expressed beliefs "I am not as serious as others with the disease" and "I feel well without treatment". ${ }^{610}$

The concept of personal control has become widely accepted as being an important aspect of health care. ${ }^{16}$ Three aspects of perceived control have been defined: (1) internal locus of control (the extent to which individuals believe that they can determine their disease progression/outcome), (2) chance locus of control (the belief that factors outside of a person's control such as luck, chance, or fate can influence their health), and (3) powerful others locus of control (the belief that other people such as doctors, nurses, or family members can influence health outcome). Patients on renal dialysis who believed that they had control over the management of their own disease (internal locus of control) adhered to their treatment to a greater extent than those who believed that the course of their disease was outside of their control (external beliefs). Patients who believed that health professionals were responsible for their health also reported higher levels of adherence to treatment. ${ }^{17}$

This study aimed to evaluate whether health beliefs or perceived control influence adherence to treatments in adults with cystic fibrosis. In the context of the Health Belief Model the work assessed the relationships between reported adherence to treatments and (1) the patients' perceived disease severity concerning their past, present and future health, (2) the extent to which they were concerned or worried about having cystic fibrosis, and (3) perceived vulnerability for recurrent infections. The relationship between reported treatment adherence and the patients' perception of where (or with whom) the responsibility and control lay for the management of their disease was also examined.

\section{Methods}

SUBJECTS

Sixty seven consecutive patients who regularly attended the Manchester Adult Cystic Fibrosis Unit were invited to take part in the study.

\section{INTERVIEW}

All patients took part in the study whilst they were clinically stable. The patient was guided through the questionnaires by a psychologist. In order to minimise the "faking good effect" subjects were administered the questionnaires away from hospital wards and clinics and confidentiality was assured.

\section{QUESTIONNAIRES}

The Manchester Adult Cystic Fibrosis Compliance Questionnaire was developed to measure the rates of adherence to treatments and medical advice, the reasons for non-adherence, and the patients' perception of their level of compliance. These preliminary data can be found elsewhere. ${ }^{6}$ For the purpose of this study only the rates of adherence to the four treatments (physiotherapy, pancreatic enzymes, vitamins, and exercise therapy) were incorporated into the analyses. The degree of reported adherence with physiotherapy was measured on a six point scale ("I never do my physiotherapy" $=0$ to "I do my physiotherapy once/ twice each day" $=5$ ). The extent of adherence with enzyme (and vitamin) treatment was measured on a four point scale ("I never take enzymes" $=0$ to "I always take enzymes as prescribed" $=3$ ). The type and frequency of exercise engaged in by each patient was assessed (independently by the physiotherapist and the physician) as to whether the exercise constituted a beneficial level for that particular patient.

Five subscales from the Health Perception Questionnaire $^{18}$ which are all components of the Health Belief Model were employed. These were past, current, and future health, susceptibility to infections, and worry about the disease. The Health Locus of Control Scale ${ }^{19}$ is comprised of three subscales which measure (1) the extent to which individuals perceive themselves to be in control of their own health, (2) the degree to which they believe their health is controlled by health professionals or family, and (3) the influence of chance factors.

\section{STATISTICAL ANALYSES}

The Statistical Package for the Social Sciences (SPSS for Windows) was employed for the data management and analyses.

It would be unrealistic to expect patients to comply with treatments absolutely, particularly with physiotherapy. Our classification of adherence is therefore less rigid. The data were collapsed to form three adherence groups: (1) adherent ("I do my physiotherapy once/twice each day, I occasionally miss my physiotherapy", $n=31$ ), (2) partially adherent ("I often miss one/two days, I often miss many days", $\mathrm{n}=10$ ), and (3) non-adherent ("I never do my physiotherapy, I only do my physiotherapy when ill", $n=19$ ).

Similarly, the enzyme and vitamin data were broken down into adherent ("I always take enzymes $(n=45) /$ vitamins $(n=27)$ as prescribed"), partially adherent ("I usually take enzymes $(n=8) /$ vitamins $(n=9)$ as prescribed"), and non-adherent groups ("I sometimes/never take enzymes $(\mathrm{n}=7) /$ vitamins $(\mathrm{n}=$ 24) as prescribed"). These data were analysed using analyses of variance and independent $t$ tests. 
Table 1 Distribution of scores for the Health Perception and Health Locus of Control (HLOC) subscales

\begin{tabular}{lrlr}
\hline Subscale & Mean & SD & Range \\
\hline Past health & 9.22 & 3.56 & $3-15$ \\
Current health & 27.72 & 5.71 & $11-44$ \\
Future health & 11.00 & 3.76 & $4-20$ \\
Susceptibility & 10.75 & 3.28 & $5-17$ \\
Worry & 10.30 & 3.18 & $4-19$ \\
HLOC (internal) & 25.07 & 4.34 & $15-35$ \\
HLOC (chance) & 24.17 & 5.07 & $13-33$ \\
HLOC (powerful others) & 24.31 & 4.63 & $12-35$ \\
\hline
\end{tabular}

For exercise therapy patients were described as either adherent (exercising to a beneficial level, $\mathrm{n}=44$ ) or non-adherent (not exercising to a beneficial level, $n=11$ ). Five patients were not prescribed exercise therapy because of the severity of their disease. The independent $t$ test was employed for the comparison of Health Perception and Health Locus of Control subscale scores between the exercise groups. Pearson correlations were performed between percentage predicted forced expiratory volume in one second $\left(\mathrm{FEV}_{1}\right)$ and scores on the psychometric scales.

\section{Results}

Sixty patients (35 men) agreed to take part in the study and constituted approximately half of the total number of patients who attended the cystic fibrosis centre. The mean age of the sample was 20.98 years (range 16-44). The mean $\mathrm{FEV}_{1}$ percentage predicted was $59.4 \%$ (range $10-103 \%$ ) indicating a wide range of disease severity. All study population means and percentages were representative of the total population of the cystic fibrosis unit.

The scores on both the Health Perception and Health Locus of Control subscales were reasonably normally distributed so mean values are presented throughout the data analyses. The range of scores and mean values for each subscale were similar to those reported in other chronic disease populations (table 1). ${ }^{2021}$ No significant correlations were observed between $\mathrm{FEV}_{1}$ percentage predicted or age and scores on the Health Perception and Health Locus of Control subscales, nor were there associations between percentage predicted $\mathrm{FEV}_{1}$, age, or sex and the level of reported adherence to the four treatment regimens.

\section{ADHERENCE AND PERCEIVED HEALTH}

There were no differences between the adherence groups (adherent, partially adherent and non-adherent) for physiotherapy, exercise, enzyme and vitamin taking with regard to scores on the perceived past, current, and future health scales or the susceptibility to illness scale. The amount of worrying about the disease, however, discriminated patients on the basis of adherence. Patients who reported themselves adherent to their physiotherapy worried about having cystic fibrosis to a greater extent than patients who were non-adherent (adherent $=13.8$, non-adherent $=7.5$, difference $=6.3,95 \%$ CI 1.48 to $5.98, \mathrm{p}<0.002$ ) or partially adherent with their physiotherapy regimen (adherent $=13.8$, partially adherent $=$ 9.6, difference $=4.2,95 \%$ CI 0.02 to 4.64 , $\mathrm{p}<0.05)$. Those adherent with their enzyme medication had higher levels of worry than non-adherent subjects (adherent $=14.5$, nonadherent $=8.1$, difference $=6.4,95 \%$ CI 1.32 to $5.86, \mathrm{p}<0.001)$. Patients who were adherent or partially adherent with their vitamin regimen worried more about their cystic fibrosis than
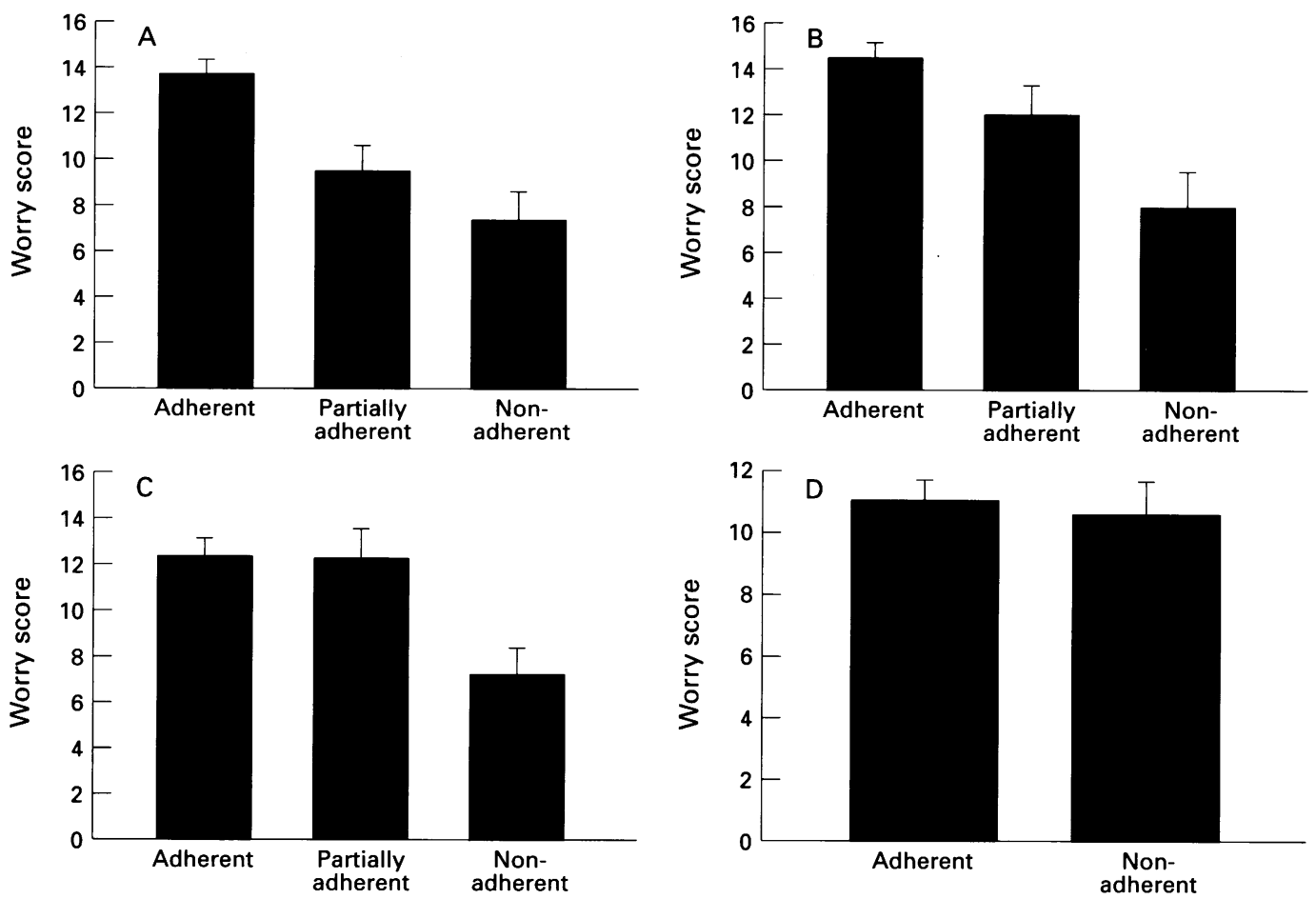

Figure 1 Mean worry scores for the adherence groups to (A) physiotherapy, (B) enzymes, (C) vitamins, and (D) exercise regimens. 

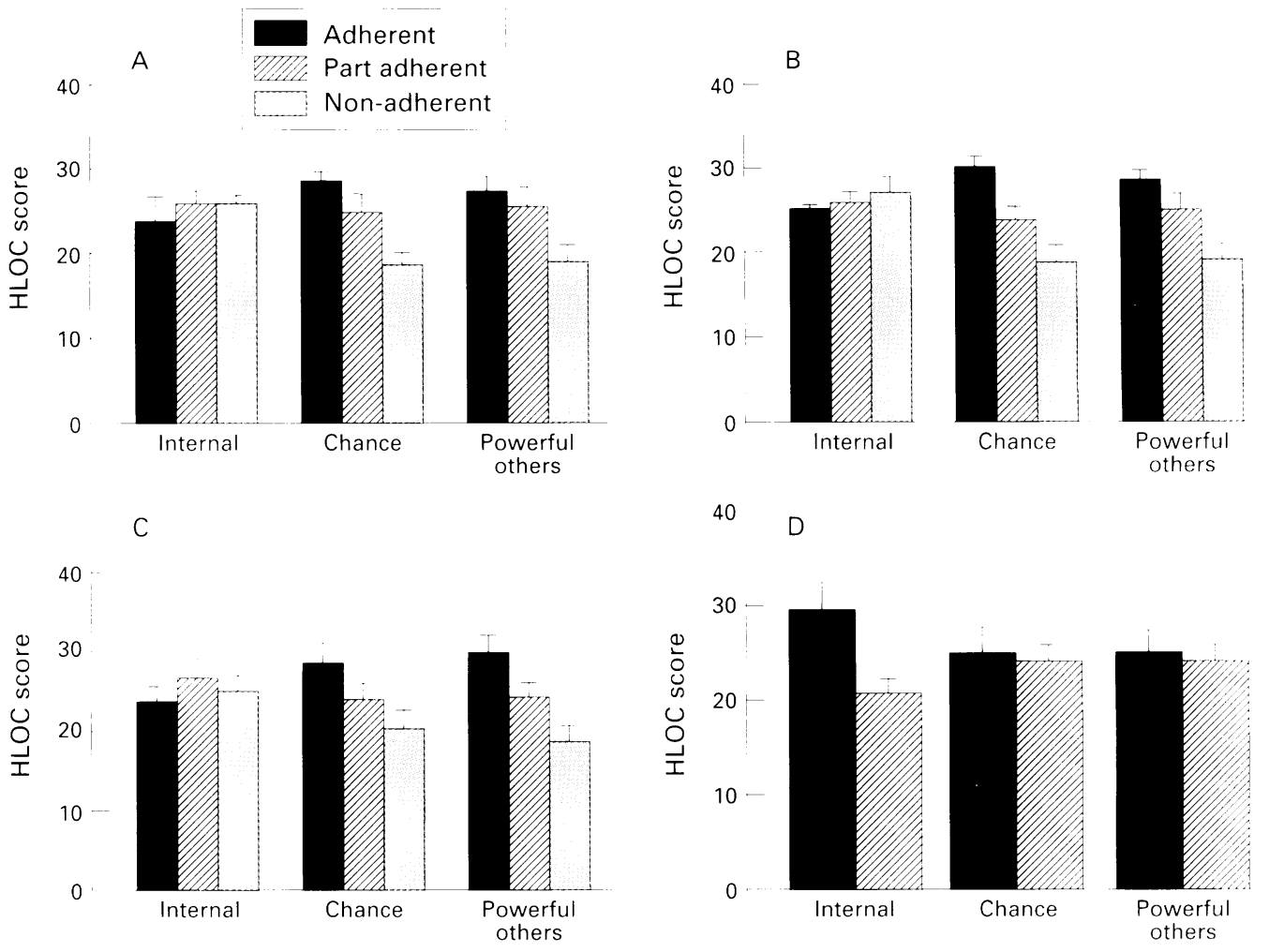

Figure 2 Mean Health Locus of Control scores for the adherence groups to (A) physiotherapy, (B) ensimes, (C) vitamins, and (D) exercise regimens. Internal control indicates the extent to which patients perceive themscless to be in control of their cystic fibrosis; the chance scale reflects the degree to which patients believe that chance factors such as fate and luck influence their health; and the powerful others scale measures the extent to which they believe thit disease is controlled by health professionals and or family.

those who were non-adherent (adherent $=12.3$, non-adherent $=7.2$, difference $=5.1,95 \% \mathrm{CI}$ 1.59 to $6.02, \mathrm{p}<0.001)$. No difference between the exercise adherent and non-adherent groups was found regarding their level of worry (adherent $=11.1$, non-adherent $=10.5$, difference $=0.6,95 \% \mathrm{CI}-2.26$ to 2.46 ) (fig 1 ).

ADHERENCE AND PERCEIVED CONTROL

Scores on the internal locus of control scale were similar for all patients regardless of their level of adherence to physiotherapy or enzyme and vitamin medication. Similar patterns of results emerged for both the chance and powerful others scales. Patients adherent or partially adherent to their physiotherapy believed that factors outside of their control and powerful others (health professionals/family members) were responsible for their health to a greater extent than patients non-adherent with their physiotherapy (chance, adherent $=28.7$, nonadherent $=18.8$, difference $=9.9,95 \%$ CI 1.47 to $12.12, \mathrm{p}<0.001$; powerful others, adherent $=$ 27.6, non-adherent $=19.4, \quad$ difference $=8.2$, $95 \%$ CI 1.17 to $9.13, \mathrm{p}<0.001$; chance, partially adherent $=25.1$, non-adherent $=18.8$, difference $=6.3,95 \%$ CI 0.47 to 9.53 , p $<0.03$; powerful others, partially adherent $=25.9$, nonadherent $=19.4$, difference $=6.5,95 \%$ CI 0.81 to $7.73, \mathrm{p}<0.05)$. Patients adherent with their enzyme medication scored higher on the chance scale than those who were partially adherent or non-adherent (adherent $=30.0$, partially adherent $=23.7$, difference $=6.3,95 \%$ CI 1.20 to $9.22, \mathrm{p}<0.01 ;$ adherent $=30.0$, non-adherent $=$
18.7, difference $=11.3,95 \%$ CI 1.83 to 14.75 , $\mathrm{p}<0.001)$. Adherent and partially adherent patients believed that others controlled their health to a greater extent than those who were non-adherent (adherent $=28.5$, non-adherent $=$ 19.1, difference $9.4,95 \%$ CI 1.3 to 11.63 , $\mathrm{p}<0.001$; partially adherent $=25.1$, non-adherent $=19.1$, difference $=6.0,95 \%$ CI 0.46 to $8.98, \mathrm{p}<0.05)$. Those patients adherent with their vitamin regimen believed that chance factors and powerful others influenced their cystic fibrosis to a greater degree than non-adherent or partially adherent patients (chance, adherent $=28.2, \quad$ non-adherent $=20.3, \quad$ difference $=7.9,95 \%$ CI 1.53 to $10.86, p<0.002$; powerful others, adherent $=29.8$, non-adherent $=18.9$, difference $=10.9,95 \%$ CI 2.17 to $14.29, \mathrm{p}<0.001$; powerful others, partially adherent $=24.2$, non-adherent $=18.9$, difference $=5.3,95 \%$ CI 0.33 to $8.71, \mathrm{p}<0.05)$. In contrast, patients who were adherent with exercise therapy believed that they were in control of their own health to a greater extent than non-adherent patients (adherent $=29.4$, non-adherent $=20.7$, difference $=8.7,95 \% \mathrm{CI}$ 1.59 to $12.55, \mathrm{p}<0.003$ ) (fig 2 ).

\section{Discussion}

The Health Belief Model was only partially supported by the findings of this study. Theoretically, the relationship between perceived severity and adherence should be a positive one, but no difference emerged between the adherence groups (reported level of adherence) regarding perceived past, present or future 
health. In contrast, other researchers have found that perceived severity of illness predicts reported treatment adherence. ${ }^{1415}$ For life threatening diseases or conditions with a shortened life expectancy the relationship between perceived severity and adherence may be more complex than the simplistic positive linear association assumed by the Health Belief Model. If the level of perceived severity becomes too great some individuals may avoid perceptions and behaviours which compel them to acknowledge the seriousness of the situation. This notion is supported by previous work which demonstrated that patients with the most serious cystic fibrosis $\left(\mathrm{FEV}_{1}<40 \%\right.$ predicted) reported their health to be "above/well above average" compared with others with cystic fibrosis even though they had been assessed for and were awaiting heart-lung transplantation. ${ }^{11}$ Similarly, in voluntary screening for Tay-Sachs disease and tuberculosis those patients who perceived the seriousness of the disease to be extremely high refused radiographic screening. ${ }^{2223}$ For many patients avoidance or denial may be an adaptive way of coping with cystic fibrosis. There is evidence that these coping strategies have a protective effect for individuals on coronary care units ${ }^{24}$ but their value remains to be evaluated as a way of coping with cystic fibrosis. Conversely, minimising the seriousness of the disease may become harmful if it diminishes adherence to treatment regimens.

The Health Belief Model assumes that perceived susceptibility to recurrent ill health would be associated with greater adherence but our results do not lend support to this assumption. Subsequently it has been hypothesised that perceived disease severity/susceptibility may be related to adherence in an inverted $U$ shaped maner, ${ }^{22325}$ adherence being low when severity is perceived as either low or high and greatest when severity is seen as moderate. It is possible that, although individuals may acknowledge the seriousness of their disease and their vulnerability to recurrent health problems, this is not sufficient to influence adherence to treatments. This work has clearly demonstrated that only if they are concerned/worried about their cystic fibrosis will they be motivated to follow their treatment regimens appropriately. Those patients who reported high levels of worry about their condition adhered to their physiotherapy, enzyme, and vitamin therapies to a greater extent than those patients who were not unduly concerned about their disease. Similar findings have emerged from a study regarding breast selfexamination. Perceived susceptibility to breast cancer or perceived disease severity were not related to the frequency of self-examination, whereas health concern predicted self-examination. ${ }^{25}$

Beliefs about control over one's health are a salient influence on treatment adherence in adults with cystic fibrosis. Theoretically, patients with internal control beliefs would be expected to take control of their own disease management and thereby adhere to treatments to a greater extent than those who believe that chance factors or powerful others are responsible for their health outcomes. In contrast to this assumption, this work has shown that external control beliefs (both chance factors and health professionals/family) were associated with higher levels of reported adherence to physiotherapy, enzyme, and vitamin regimens. Other studies have noted greater levels of adherence in those who rely on family members and health professionals. Patients who believed that it was important to follow doctors' instructions and those who had faith in the benefits of the treatment were more likely to adhere to treatments. ${ }^{141526}$ It was possible that those with external control beliefs may have reported higher levels of adherence because they worried more about their cystic fibrosis. No significant correlation emerged between external control beliefs scores and worry scores, indicating that these constructs were independent.

These findings are not necessarily incongruent with the assumption that those patients who believe that they are in control of their health (internals) would take responsibility for their own disease management. Those with internal beliefs may make a deliberate decision not to adhere to their treatment recommendations completely if they perceive themselves to be well and, in this respect, control their own disease management. Alternatively, their partial or non-adherence to treatments may be the product of a rational decision enabling a balance between their treatment regimens and their quality of life. In contrast to other findings, those who believe that they are responsible for their own management (internals) adhere to exercise therapy to a greater degree than patients with external beliefs. Given that exercise was the only therapy in which higher levels of adherence were not related to worrying about having cystic fibrosis, it seems that exercise is perceived differently to other forms of treatment. Its acceptance as a "normal" activity for young adults in comparison with physiotherapy and medication taking may partly explain why exercise is more popular.

This study has evaluated treatment adherence using self-report methodology. Various direct (blood or urine assays) and indirect (pill counts, mechanical devices, physician estimates and self-report) methods have previously been used to assess treatment adherence. Each method is not without problems and disadvantages. ${ }^{27-29}$ There is evidence of overreporting self-administered oral medication compared with pill counting and blood or urine assays. ${ }^{30}$ In cystic fibrosis adherence to "behavioural" treatments (physiotherapy and exercise) also need to be evaluated. Since the relationship between the rate of decline in clinical status and levels of treatment adherence in individuals is unclear, we currently have to rely on measures of reported adherence against which there are no appropriate objective measures to validate the data. Although there is a need to be mindful of inflated self-reported adherence rates, it is encouraging that those who report non-adherence rarely $\operatorname{lie}^{29}$ and only 
this group responded consistently to interventions. $^{2}$

If we expect all patients to be totally adherent we will always be disappointed. Considerations regarding the balance between adherence to treatments and the patient's quality of life need to be made and a non-judgemental approach needs to be taken. ${ }^{31}$ These findings have raised important ethical and complex questions concerning the management of non-adherence. If improving adherence means ensuring that patients face up to the clinical reality of their condition, more harm than benefit may be created. Family members also share the patient's health perceptions. Worrying about cystic fibrosis is a major motivating factor for adherence yet it would be unethical to foster an atmosphere of worry and concern. The identification of beneficial coping strategies for both psychological well being and disease progression would improve our ability to manage non-adherence successfully.

1 The Cystic Fibrosis Trust. UK Cystic Fibrosis Survey Report. 1993 .

2 Sackett DL, Snow JC. The magnitude of compliance and non-compliance. In: Haynes RB, Taylor DW, Sacket DL, eds. Compliance in health care. Baltimore: John Hopkins eds. Compliance in health care.

3 Stanway L, Lambie DG, Johnson RH. Non-compliance with anticonvulsant therapy as a cause of seizures. NZ Med f 1985;98:150-2.

4 Christiaanse M, Lavigne J, Lerner C. Psychosocial aspects of compliance in children and adolescents with asthma. $\mathscr{f}$ Devel Behav Pediatr 1989;10:75-80.

5 Brown J, Fitzpatrick R. Factors influencing compliance with dietary restrictions in dialysis patients. 7 Psychosom Res 1988;32:191-6.

6 Abbott J, Dodd M, Bilton D, Webb AK. Treatment compliance in adults with cystic fibrosis. Thorax 1994;49. $115-20$.

7 Passero MA, Remor B, Salomon J. Patient-reported com pliance with cystic fibrosis therapy. Clin Pediatr 1981;20. 264-8

8 Czajkowski D, Koocher G. Medical compliance and coping with cystic fibrosis. F Child Psychol Psychiatry 1987;23: 311-9.

9 Giess S, Hobbs S, Hannersley-Maercklein G, Kramer J, Henley M. Psychosocial factors related to perceived com- pliance with cystic fibrosis treatment. $\mathscr{f}$ Clin Psychol 1992; 48:99-103.

10 Tebbi C, Cummings $M$, Zevon $M$, Smith L, Richards $M$, Mallon J. Compliance of pediatric and adolescent cancer patients. Cancer 1986;58:1179-84

11 Abbott J, Dodd M, Webb AK. Different perceptions of disease severity and self care between patients with cystic fibrosis, their close companion, and physician. Thorax 1995;50:794-6.

12 Gudas LJ, Koocher GP, Wypij D. Perceptions of medical compliance in children and adolescents with cystic fibrosis. Devel Behav Pediatr 1991;12:236-42.

13 Becker M. The health belief model and personal health behaviour. Thorofare, NJ: Slack, 1974.

14 Kirscht JP, Rosenstock IM. Patient adherence to antihypertensive medical regimens. 7 Community Health 1977 ; 3:115-24.

15 Hartman PE, Becker MH. Non-compliance with prescribed regimen among chronic hemodialysis patients. Dialysis Transplant 1978;7:978-85.

16 Steptoe A, Appels A. Stress, personal control and health. Chichester: Wiley, 1989.

17 Poll I, Kaplan A. Locus of control and adjustment to chronic haemodialysis. Psychol Med 1980;10:153-7.

18 Ware JE. Scales for measuring health perceptions. Health Services Res 1976;6:161-70

19 Wallston BS, Wallston KA, Kaplan GD, Mades SA. Development and validation of the health locus of control scale. F Consult Clin Psychol 1976;44:580-5.

20 Harkapaa K, Jarvikoski A, Mellin G, Hurri M, Luoma J. Health locus of control beliefs and psychological distress as predictors for the outcome in low back pain patients: results of a three month follow-up of a controlled interresults of a three month follow-up
vention study. Pain 1991;46:35-41.

21 Partridge $C$, Johnston M. Perceived control and recovery from physical disability: measurement and prediction. $\mathrm{Br}$ from physical disability: mea

22 Becker M, Kabak M, Rosenstock I, Ruth M. Some influences of public participation in a genetic screening program. $\mathcal{f}$ Community Health 1975;1:3-14.

23 Hochbaum G. Why people seek diagnostic x-rays. Public Health Rep 1956;71:377-80.

24 Hackett TP, Cassem NH, Wishnie HA. The coronary care unit: an appraisal of its psychologic hazards. $N$ Engl $\mathcal{F} \mathrm{Med}$ 1968;279:1365-70

25 Champion V. Use of the health belief model in determining frequency of breast self-examination. Res Nurs Health 1985;8:373-9.

26 Becker MH, Nathanson CA, Drachman RH. Mothers' health beliefs and children's clinic visits: a prospective study. F Community Health 1977;3:125-35.

27 Wright EC. Non-compliance - or how many aunts has Matilda? Lancet 1993;342:909-13.

28 Litt IF, Cuskey WR. Compliance with medical regimens during adolescence. Pediatr Clin North Am 1980;27:3-15.

29 Epstein LH, Cluss PA. A behavioural medicine perspective on adherence to long-term medical regimens. $\mathcal{F}$ Consult Clin Psychol 1982;50:950-71.

30 Ley P. Satisfaction, compliance and communication. $\mathrm{Br} f$ Clin Psychol 1982;21:241-54.

31 Lask B. Non-adherence to treatment in cystic fibrosis. $7 R$ Soc Med 1994;87:25-8. 